\title{
Letter: Psycho-Physiological Responses to a 4-Month High-Intensity Interval Training-Centered Multidisciplinary Weight-Loss Intervention in Adolescents with Obesity ( $\mathrm{J}$ Obes Metab Syndr 2020;29:292-302)
}

Hyung Jin Choi*

Department of Biomedical Sciences, Anatomy and Cell Biology, Seoul National University College of Medicine, Seoul, Korea

Pediatric obesity is a major public health burden. ${ }^{1}$ Nutritional management ${ }^{2}$ and exercise ${ }^{3}$ promotion are a main axis of behavioral intervention for pediatric obesity. One of the major obstacles in pediatric obesity is to motivate children and adolescents to adhere to treatment. ${ }^{4}$ Therefore, psychology underlying pediatric obesity is an important research area, although not many researches have investigated this issue directly and intensively.

The paper "Psycho-physiological responses to a 4-month highintensity interval training-centered multidisciplinary weight-loss intervention in adolescents with obesity" ${ }^{\prime 3}$ investigated the impact of 16-week high-intensity interval training on psychological responses and their association with changes in intervention outcomes (body weight, body composition, and aerobic capacity) with 56 adolescents ( 28 girls and 28 boys; aged from 11 to 17 years; mean body mass index (BMI) z-score, 2.34; BMI percentile, 98.58). Health-related quality of life (HR-QOL), health perception (HP), and physical self-perception (PSP) were used for assessments. Among anthropometric results, there were significant improvements in body weight ( $92.6 \pm 18.9$ to $85.9 \pm 16.2 \mathrm{~kg})$,
BMI $\left(35.0 \pm 4.8\right.$ to $\left.32.1 \pm 4.5 \mathrm{~kg} / \mathrm{m}^{2}\right), \mathrm{z}-\mathrm{BMI}(2.3 \pm 0.3$ to $2.1 \pm 0.3)$, and fat mass percentage $(36.0 \% \pm 9.1 \%$ to $30.4 \% \pm 7.8 \%)$. Among psychological results, there were significant improvements in physical functioning, physical limitation, general health, bodily pain, physical condition, adiposity, alimentation, general health, perceived general health, self-perceived coordination, endurance, activity, global self-concept, and appearance. The authors reported that the multidisciplinary weight loss intervention improved HR-QOL, HP, and PSP. In addition, physical HR-QOL but not mental HR-QOL domains of HP and PSP were associated with weight reduction. The results are interesting. However, in my opinion, some additional information will increase reader understanding.

Psychological support (1/month) was included in this multidisciplinary weight loss intervention. Since this study reported remarkable improvement on psychological responses, more details on the detailed components of psychological support intervention would help readers to design an intervention program equipped with appropriate psychological components. The actu- 
al contents for a 4-month psychological program, including training for the psychological interventionist (or coach), qualification or specialties of the interventionist (e.g., doctors, nurses, or psychologists), duration of intervention, involvement of psychological theory ${ }^{4}$ (e.g., cognitive behavioral therapy, ${ }^{5}$ transtheoretical model of change or motivational interviewing), involvement of caregivers (e.g., parents), and involvement of personalized components based on personal characteristics (e.g., baseline survey results) would be very helpful for readers.

Adherence, engagement, and motivation toward the intervention program are other major issues in lifestyle modification intervention. When applying lifestyle modification interventions to the adolescent population, these critical issues are related to low effectiveness of the intervention and high dropout. ${ }^{4}$ To improve treatment effectiveness, it is critical to monitor the motivation of participants to provide adequate strategies that can enhance motivation for lifestyle changes. ${ }^{4}$ Among the 56 participants, those with higher motivation and higher engagement would have better improvement in both psychological components and anthropometric components than those with lower motivation. Results regarding any indices related to engagement (e.g., number of visits or adherence to the exercise program) would provide additional insight into the role of engagement (and motivation) with the intervention efficacy. Another issue is recruitment. The present paper described that, "adolescents with obesity were recruited from an inpatient weight-management intervention." Since the adolescents and their caregivers visited the clinic for treatment, their motivation would be higher than that of a general population or a participant screened from school (who had no intention to visit clinic). A brief description of the motivation status of the participants would be of much help.

Weight regain after intervention is a major bottleneck for obesity therapy. Since the present study provided good evidence that these adolescents improved their psychological aspects, it is likely that this multidisciplinary intervention produced fundamental lifestyle change. The sustained effects could be provided by longterm follow-up data after the intervention.

Digital intervention or a digital platform is a novel powerful method in the field of obesity intervention. Since the authors are experts in the field of pediatric obesity intervention, it would be helpful if the authors could provide comments or suggestions on application of digital methods for multidisciplinary intervention for pediatric obesity.

The present study in Journal of Obesity and Metabolic Syndrome makes an important contribution to the field of pediatric obesity and multidisciplinary intervention for obesity. These data provide direct evidence of improvement in psychological characteristics and the role of psychological components in anthropometric outcomes regarding multidisciplinary intervention for obesity.

\section{CONFLICTS OF INTEREST}

Hyung Jin Choi has been the Editor of Journal of Obesity and Metabolic Syndrome since 2007. However, he was not involved in peer reviewer selection, evaluation, or decision processes of this article. No other potential conflicts of interest relevant to this article were reported.

\section{REFERENCES}

1. Dimitri P. The impact of childhood obesity on skeletal health and development. J Obes Metab Syndr 2019;28:4-17.

2. Kim J, Lim H. Nutritional management in childhood obesity. J Obes Metab Syndr 2019;28:225-35.

3. Khammassi M, Miguet M, Julian V, Cardenoux C, Boirie Y, Duclos M, et al. Psycho-physiological responses to a 4-month high-intensity interval training-centered multidisciplinary weightloss intervention in adolescents with obesity. J Obes Metab Syndr 2020;29:292-302.

4. Woo S, Park KH. Motivating children and adolescents in obesity treatment. J Obes Metab Syndr 2020;29:260-9.

5. Castelnuovo G, Pietrabissa G, Manzoni GM, Cattivelli R, Rossi A, Novelli M, et al. Cognitive behavioral therapy to aid weight loss in obese patients: current perspectives. Psychol Res Behav Manag 2017;10:165-73. 